\title{
Operator Method of Nonstationary Temperature Field Calculation in Environment With an Isolated Cylindrical Pipe
}

\author{
Voldemārs Barkāns ${ }^{1 *}$, Gints Turlajs ${ }^{2}$, Aivars Cers ${ }^{3}$, Roberts Veselauskis ${ }^{4}$, Daniels Turlajs ${ }^{5}$, Sigurds Jaundālders ${ }^{6}$ \\ ${ }^{1}$ Latvian Maritime Academy, Latvia ${ }^{2}$ Smart Continent LV Ekoterm SIA, Latvia ${ }^{3,4}$ Latvenergo AS, Latvia \\ ${ }^{5,6}$ Riga Technical University, Latvia
}

\begin{abstract}
The following research is important for the understanding of thermal conductivity processes in the setting typical for thermal pumps and for bringing calculations nearer to the real situation. Considering a non-stationary process, the temperature field is described in the polar coordinate system by using Laplace's equation and corresponding mixed-type boundary data. The solution was obtained by the Laplace transform method, applying an integral function of complex variables. The inverse Laplace transform and the original temperature are expressed as an integral. For the integration, a closed contour, which rules out branching and provides an integral of an analytic function, is employed. Cauchy's theorem is applied to the calculations. As a result, indefinite integrals have been derived for temperature estimation in the heat pipe coating and the surrounding environment, depending on the fluid temperature within the heat pipe.
\end{abstract}

Keywords - Heat pumps, Laplace transform, thermal conductivity.

\section{INTRODUCTION}

The following research is important for the understanding of thermal conductivity processes in the setting typical for a modern renewable heat source with a further development perspective, namely, thermal pumps. The understanding of the limitations of the thermal pumps technology also has very important economic implications. Imperfections in thermal conductivity may change the efficiency of thermal pumps technology several times and should be taken into account when taking investment decisions. Since the general principle of heat transfer in such circumstances is similar in other settings as well, the following research is also useful with other applications. The heat flows currently calculated may significantly differ from the actual values, a difference that is very well observable between theoretical calculations of ground heat pump productivity and actually observed heat flows. The temperature field parameter methodology developed within this study is needed to determine the heat flow with a greater degree of accuracy.

\section{DESCRIPTION OF NON-STATIONARY TEMPERATURE FIELD}

A non-stationary temperature field $T(r, \tau)[\mathrm{K}]$ in environment with a cylindrical pipe (Fig. 1), which is filled with a special fluid, at a constant temperature $T_{0}[\mathrm{~K}]$, can be described [10] with a thermal conductivity equation in polar coordinates:

$$
\frac{\partial T(r, \tau)}{\partial \tau}=a\left(\frac{\partial^{2} T(r, \tau)}{\partial r^{2}}+\frac{1}{r} \frac{\partial T(r, \tau)}{\partial r}\right) ; r \geq r_{0} ; \tau \geq 0,
$$

where

$$
\partial T(r, \tau)=\left\{\begin{array}{l}
T_{0}(r, \tau), \text { if } 0 \leq r \leq r_{0} ; \text { fluid in pipe; } \\
T_{1}(r, \tau), \text { if } r_{0} \leq r \leq r_{1} ; \quad \text { in pipe housing; } \\
T_{2}(r, \tau), \text { if } r_{1} \leq r \leq r_{2} ; \quad \text { in pipe insulation; } \\
T_{3}(r, \tau), \text { if } r \leq r_{2} ; \quad \text { in soil. }
\end{array}\right.
$$

$a$ - thermal diffusion coefficient, $\mathrm{m}^{2} / \mathrm{s}$;

$$
a=\left\{\begin{array}{llr}
a_{1}, & \text { if } & r_{0} \leq r \leq r_{1} ; \\
a_{2}, & \text { if } & r_{1} \leq r \leq r_{2} \\
a_{3}, & \text { if } & r \geq r_{2} .
\end{array}\right.
$$

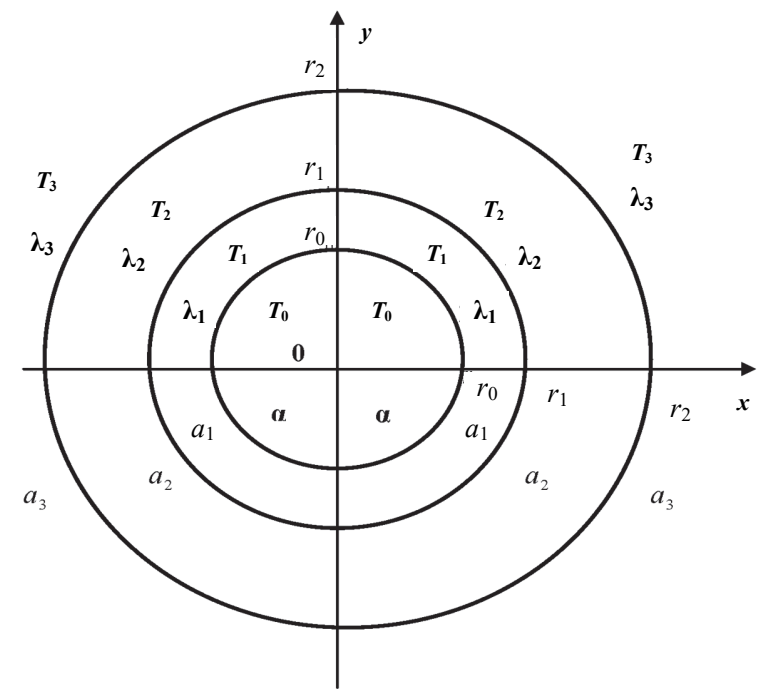

Fig. 1. Cross-section of a pipe. 
Equation (1) is overwritten using (2) and (3):

$$
\frac{\partial T_{i}(r, \tau)}{\partial \tau}=a_{i}\left(\frac{\partial^{2} T_{i}(r, \tau)}{\partial r^{2}}+\frac{1}{r} \frac{\partial T_{i}(r, \tau)}{\partial r}\right), i=1 ; 2 ; 3
$$

On boundary $r=r_{0}$, heat exchange between the fluid and the pipe housing proceeds in accordance with Newton's law:

$$
\lambda_{1} \frac{\partial T_{1}\left(r_{0}, \tau\right)}{\partial r}=-\alpha\left[T_{0}-T_{1}\left(r_{0}, \tau\right)\right],
$$

where $\alpha$ - heat transfer coefficient, $\mathrm{W} /\left(\mathrm{m}^{2} \mathrm{~K}\right)$;

$T_{0}$ - fluid temperature in pipe, $\mathrm{K}$.

On boundary $r=r_{1}$, the temperatures and heat flows must be equal:

$$
\begin{gathered}
T_{2}\left(r_{1}, \tau\right)=T_{1}\left(r_{1}, \tau\right) \\
\lambda_{2} \frac{\partial T_{2}\left(r_{1}, \tau\right)}{\partial r}=\lambda_{1} \frac{\partial T\left(r_{1}, \tau\right)}{\partial r},
\end{gathered}
$$

where $\lambda_{1}$ - pipe housing heat transfer coefficient, $\mathrm{W} /(\mathrm{m} \mathrm{K})$.

On boundary $r=r_{2}$, the temperatures and heat flows must be equal:

$$
\begin{gathered}
T_{2}\left(r_{2}, \tau\right)=T_{3}\left(r_{2}, \tau\right) ; \\
\lambda_{2} \frac{\partial T_{2}\left(r_{2}, \tau\right)}{\partial r}=\lambda_{3} \frac{\partial T\left(r_{2}, \tau\right)}{\partial r},
\end{gathered}
$$

where $\lambda_{2}$ - pipe insulation heat transfer coefficient, $\mathrm{W} /(\mathrm{m} \mathrm{K})$; $\lambda_{3}$ - soil heat transfer coefficient, $\mathrm{W} /(\mathrm{m} \mathrm{K})$.

Temperature of the starting moment $\tau=0$ :

$$
T_{i}(r, 0)=T_{0 i} ; \quad i=1,2,3
$$

At a large distance from the pipe axis, the temperature is constant:

$$
T_{3}(\infty, \tau)=T_{\infty}
$$

and

$$
\frac{\partial T_{3}(\infty, \tau)}{\partial r}=0
$$

It can be assumed that

$$
T_{01}=T_{02}=T_{03}=T_{\infty} .
$$

Using dimensionless variables and parameters [1]:

$$
\frac{r}{r_{0}}=\rho ; \quad \frac{r_{1}}{r_{0}}=\rho_{1} ; \quad \frac{r_{2}}{r_{0}}=\rho_{2} ;
$$

$$
\begin{gathered}
\frac{T_{i}}{T_{0}}=\Theta_{i} ; \quad \frac{T_{0 i}}{T_{0}}=\Theta_{0 i} ; \quad \frac{T_{\infty}}{T_{0}}=\Theta_{\infty}=\Theta_{0 i} ; i=1,2,3 ; \\
\frac{a_{1}}{a_{2}}=k_{2}^{2} ; \quad \frac{a_{1}}{a_{3}}=k_{3}^{2} ; \quad \frac{\lambda_{1}}{\lambda_{2}}=\Lambda_{1} ; \quad \frac{\lambda_{2}}{\lambda_{3}}=\Lambda_{2} ; \\
F=\frac{a_{1} \tau}{r_{0}^{2}} ; \quad B i=\frac{\alpha r_{0}}{\lambda_{1}} .
\end{gathered}
$$

$\Theta(p, F)=\left\{\begin{array}{l}1, \text { if } 0 \leq \rho \leq 1 ; \text { fluid in pipe; } \\ \Theta_{1}(\rho, F), \text { if } 1 \leq \rho \leq \rho_{1} ; \text { in pipe housing; } \\ \Theta_{2}(\rho, F), \text { if } \rho_{1} \leq \rho \leq \rho_{2} ; \text { in pipe insulation; (18) } \\ \Theta_{3}(\rho, F), \text { if } \rho \leq \rho_{2} ; \text { in soil. }\end{array}\right.$

The task is solved using Laplace transformation [1]-[5]:

$$
\bar{\Theta}_{i}(\rho, p)=\int_{0}^{+\infty} e^{-p F} \Theta_{i}(\rho, F) \mathrm{d} F
$$

and temperature images are obtained:

$$
\begin{aligned}
& \bar{\Theta}_{1}(\rho, p)= \\
& =C_{1} I_{0}(\rho \sqrt{p})+C_{2} K_{0}(\rho \sqrt{p})+\frac{\Theta_{01}}{p} ; \quad 1 \leq \rho \leq \rho_{1} ; \\
& \bar{\Theta}_{2}(\rho, p)=C_{3} I_{0}\left(k_{2} \rho \sqrt{p}\right)+ \\
& \quad+C_{4} K_{0}\left(k k_{2} \rho \sqrt{p}\right)+\frac{\Theta_{02}}{p} ; \rho_{1} \leq \rho \leq \rho_{2} ; \\
& \bar{\Theta}_{3}(\rho, p)=C_{5} K_{0}\left(k_{3} \rho \sqrt{p}\right)+\frac{\Theta_{03}}{p} ; \rho_{2} \leq \rho<+\infty
\end{aligned}
$$

where $I_{0}(z)$ and $K_{0}(z)$ - modified zero-order Bessel (McDonald) functions of the first and second type [6]-[9].

Using the boundary conditions, the coefficients of the temperature images of the obtained expression are calculated (20)-(22):

$$
\begin{aligned}
& \bar{\Theta}_{1}(\rho, p)=\frac{\Theta_{01}}{p}+\frac{\Delta C_{1}(\sqrt{p})}{\Delta(\sqrt{p})} I_{0}(\rho \sqrt{p}) \\
& +\frac{\Delta C_{2}(\sqrt{p})}{\Delta(\sqrt{p})} K_{0}(\rho \sqrt{p}) ; \\
& \bar{\Theta}_{2}(\rho, p)=\frac{\Theta_{02}}{p}+\frac{\Delta C_{3}(\sqrt{p})}{\Delta(\sqrt{p})} I_{0}\left(k_{2} \rho \sqrt{p}\right) \\
& +\frac{\Delta C_{4}(\sqrt{p})}{\Delta(\sqrt{p})} K_{0}\left(k_{2} \rho \sqrt{p}\right) ;
\end{aligned}
$$




$$
\bar{\Theta}_{3}(\rho, p)=\frac{\Theta_{03}}{p}+\frac{\Delta C_{5}(\sqrt{p})}{\Delta(\sqrt{p})} K_{0}\left(k_{3} \rho \sqrt{p}\right),
$$

where

$$
\begin{aligned}
& \Delta(\sqrt{p})=k_{2} k_{3} K_{1}(d)\left[I_{1}(b) K_{0}(c)+K_{1}(b) I_{0}(c)\right] \\
& \cdot\left[\sigma_{1}(\sqrt{p}) K_{0}(a)+\sigma_{2}(\sqrt{p}) I_{0}(a)\right] \\
& +\Lambda_{1} k_{3} K_{1}(d)\left[I_{0}(b) K_{0}(c)-K_{0}(b) I_{0}(c)\right] \\
& \cdot\left[\sigma_{1}(\sqrt{p}) K_{1}(a)-\sigma_{2}(\sqrt{p}) I_{1}(a)\right] \\
& +\Lambda_{2} k_{2}^{2} K_{0}(d)\left[I_{1}(b) K_{1}(c)-K_{1}(b) I_{1}(c)\right] \\
& \cdot\left[\sigma_{1}(\sqrt{p}) K_{0}(a)+\sigma_{2}(\sqrt{p}) I_{0}(a)\right] \\
& +\Lambda_{1} \Lambda_{2} k_{2} K_{0}(d)\left[I_{0}(b) K_{1}(c)+K_{0}(b) I_{1}(c)\right] \\
& \cdot\left[\sigma_{1}(\sqrt{p}) K_{1}(a)-\sigma_{2}(\sqrt{p}) I_{1}(a)\right] \text {, } \\
& \Delta C_{1}(\sqrt{p})=\frac{B i}{p}\left(\Theta_{01}-1\right)\left[k_{2} k_{3} K_{0}(\bar{a}) K_{1}(d)\right. \\
& \cdot\left[I_{1}(b) K_{0}(c)+K_{1}(b) I_{0}(c)\right]+\Lambda_{1} k_{3} K_{1}(\bar{a}) K_{1}(d) \\
& \cdot\left[I_{0}(b) K_{0}(c)-K_{0}(b) I_{0}(c)\right]+\Lambda_{2} k_{2}^{2} K_{0}(\bar{a}) K_{0}(d) \\
& \cdot\left[I_{1}(b) K_{1}(c)-K_{1}(b) I_{1}(c)\right]+\Lambda_{1} \Lambda_{2} k_{2} K_{1}(\bar{a}) K_{0}(d) \\
& \left.\cdot\left[I_{0}(b) K_{1}(c)+K_{0}(b) I_{1}(c)\right]\right] \text {; } \\
& \Delta C_{2}(\sqrt{p})=\frac{B i}{p}\left(\Theta_{01}-1\right)\left[-k_{2} k_{3} I_{0}(\bar{a}) K_{1}(d)\right. \\
& \cdot\left[I_{1}(b) K_{0}(c)+K_{1}(b) I_{0}(c)\right]+\Lambda_{1} k_{3} I_{1}(\bar{a}) K_{1}(d) \\
& \cdot\left[I_{0}(b) K_{0}(c)-K_{0}(b) I_{0}(c)\right]-\Lambda_{2} k_{2}^{2} I_{0}(\bar{a}) K_{0}(d) \\
& \cdot\left[I_{1}(b) K_{1}(c)-K_{1}(b) I_{1}(c)\right]+\Lambda_{1} \Lambda_{2} k_{2} I_{1}(\bar{a}) K_{0}(d) \\
& \left.\cdot\left[I_{0}(b) K_{1}(c)+K_{0}(b) I_{1}(c)\right]\right] \text {; } \\
& \Delta C_{3}(\sqrt{p})=\frac{B i}{p}\left(\Theta_{01}-1\right) \frac{\Lambda_{1}}{\rho_{1} \sqrt{p}} \\
& \cdot\left[k_{3} K_{0}(c) K_{1}(d)+\Lambda_{2} k_{2} K_{0}(d) K_{1}(c)\right] \text {; } \\
& \Delta C_{4}(\sqrt{p})=\frac{B i}{p}\left(\Theta_{01}-1\right) \frac{\Lambda_{1}}{\rho_{1} \sqrt{p}} \\
& \text { - }\left[k_{3} I_{0}(c) K_{1}(d)-\Lambda_{2} k_{2} K_{0}(d) I_{1}(c)\right] \text {; } \\
& \Delta C_{5}(\sqrt{p})=\frac{B i}{p}\left(\Theta_{01}-1\right) \frac{\Lambda_{1} \Lambda_{2}}{\rho_{1}^{2} p},
\end{aligned}
$$

where

$\bar{a}=\rho_{1} \sqrt{p} ; \quad b=k_{2} \rho_{1} \sqrt{p} ;$

$$
\begin{gathered}
c=k_{2} \rho_{2} \sqrt{p} ; \quad d=k_{3} \rho_{2} \sqrt{p} ; \\
\sigma_{1}(\sqrt{p})=\sqrt{p} I_{1}(\sqrt{p})-B i I_{0}(\sqrt{p}) \\
\sigma_{2}(\sqrt{p})=\sqrt{p} K_{1}(\sqrt{p})+B i K_{0}(\sqrt{p}) .
\end{gathered}
$$

The original temperature is expressed [1]-[3] with the following integral:

$$
\Theta_{i}(\rho, F)=\frac{1}{2 \pi j} \int_{\gamma-j \omega}^{\gamma+j \omega} \mathrm{e}^{p F} \vec{\Theta}_{i}(\rho, p) \mathrm{d} p,
$$

using formulas (23)-(25).

\section{Pipe Housing Temperature Calculation}

The original pPipe housing temperature is expressed in [1] [3] using formulas (23) and (35) with an integral as follows:

$$
\begin{aligned}
\Theta_{1}(\rho, F)= & \Theta_{0 i}+\frac{1}{2 \pi j} \int_{\gamma-j \omega}^{\gamma+j \omega} \mathrm{e}^{p F} \frac{\Delta C_{1}(\sqrt{p})}{\Delta(\sqrt{p})} I_{0}(\rho \sqrt{p}) \mathrm{d} p \\
& +\frac{1}{2 \pi j} \int_{\gamma-j \omega}^{\gamma+j \omega} \mathrm{e}^{p F} \frac{\Delta C_{2}(\sqrt{p})}{\Delta(\sqrt{p})} K_{0}(\rho \sqrt{p}) \mathrm{d} p,
\end{aligned}
$$

where $j=\sqrt{-1}$.

The calculation of complex-variable integrals is discussed in monographs [1]-[3] and publications [4], [5].

Expressing complex-variable function integrals in a realistic manner, dimensionless expression of pipe housing temperature $\left(1 \leq \rho \geq \rho_{1}\right)$ yields the following equation:

$$
\begin{aligned}
& \Theta_{1}(\rho, F)=1+\frac{2}{\pi} B i\left(\Theta_{01}-1\right) \\
& \cdot \int_{0}^{+\infty} \mathrm{e}^{-u^{2} F} \frac{\phi(u) \psi_{1}(u)-\psi(u) \phi_{1}(u)}{\phi^{2}(u)+\psi^{2}(u)} J_{0}(\rho u) \frac{\mathrm{d} u}{u}-\frac{2}{\pi} B i \\
& \cdot\left(\Theta_{01}-1\right) \int_{0}^{+\infty} \mathrm{e}^{-u^{2} F} \frac{\phi(u) \psi_{2}(u)-\psi(u) \phi_{2}(u)}{\phi^{2}(u)+\psi^{2}(u)} K_{0}(\rho u) \frac{\mathrm{d} u}{u},
\end{aligned}
$$

where

$$
\begin{aligned}
& \phi(u)=k_{2} k_{3} Y_{1}(d) \cdot U_{1}(u) \cdot S_{0}(u) \\
& +\Lambda_{1} k_{3} Y_{1}(d) \cdot U_{2}(u) S_{1}(u)+\Lambda_{2} k_{2}^{2} Y_{0}(d) \cdot U_{3}(u) \cdot S_{0}(u) \\
& +\Lambda_{1} \Lambda_{2} k_{2} Y_{0}(d) \cdot U_{4}(u) \cdot S_{1}(u) . \\
& \psi(u)=k_{2} k_{3} J_{1}(d) \cdot U_{1}(u) \cdot S_{0}(u)+\Lambda_{1} k_{3} J_{1}(d) \cdot U_{2}(u) \cdot S_{1}(u) \\
& +\Lambda_{2} k_{2}^{2} J_{0}(d) \cdot U_{3}(u) \cdot S_{0}(u)+\Lambda_{1} \Lambda_{2} k_{2} J_{0}(d) \cdot U_{4}(u) \cdot S_{1}(u) \\
& \phi_{1}(u)=k_{2} k_{3} Y_{0}\left(\bar{a}_{1}\right) Y_{1}(d) \cdot U_{1}(u) \\
& +\Lambda_{1} k_{3} Y_{1}\left(\bar{a}_{1}\right) Y_{1}(d) \cdot U_{2}(u) \Lambda_{2} k_{2}^{2} Y_{0}\left(\bar{a}_{1}\right) Y_{0}(d) \cdot U_{3}(u)
\end{aligned}
$$


$+\Lambda_{1} \Lambda_{2} k_{2} Y_{1}\left(\bar{a}_{1}\right) Y_{0}(d) \cdot U_{4}(u)$

$\psi_{1}(u)=k_{2} k_{3} Y_{0}\left(\bar{a}_{1}\right) J_{1}(d) \cdot U_{1}(u)$

$+\Lambda_{1} k_{3} Y_{1}\left(\bar{a}_{1}\right) J_{1}(d) \cdot U_{2}(u)+\Lambda_{2} k_{2}^{2} Y_{0}\left(\bar{a}_{1}\right) J_{0}(d) U_{3}(u)$

$+\Lambda_{1} \Lambda_{2} k_{2} Y_{1}\left(\bar{a}_{1}\right) J_{0}(d) \cdot U_{4}(u)$;

$\phi_{2}(u)=k_{2} k_{3} J_{0}\left(\bar{a}_{1}\right) Y_{1}(d) \cdot U_{1}(u)$

$+\Lambda_{1} k_{3} J_{1}\left(\bar{a}_{1}\right) Y_{1}(d) \cdot U_{2}(u)$

$+\Lambda_{2} k_{2}^{2} J_{0}\left(\bar{a}_{1}\right) Y_{0}(d) U_{3}(u)$

$+\Lambda_{1} \Lambda_{2} k_{2} J_{1}\left(\bar{a}_{1}\right) Y_{0}(d) \cdot U_{4}(u)$;

$\psi_{2}(u)=k_{2} k_{3} J_{0}\left(\bar{a}_{1}\right) J_{1}(d) \cdot U_{1}(u)$

$+\Lambda_{1} k_{3} J_{1}\left(\bar{a}_{1}\right) J_{1}(d) \cdot U_{2}(u)$

$+\Lambda_{2} k_{2}^{2} J_{0}\left(\bar{a}_{1}\right) J_{0}(d) U_{3}(u)$

$+\Lambda_{1} \Lambda_{2} k_{2} J_{1}\left(\bar{a}_{1}\right) J_{0}(d) \cdot U_{4}(u)$;

where

$\bar{a}_{1}=\rho_{1} u ; \quad b_{1}=k_{2} \rho_{1} u ; \quad c_{1}=k_{2} \rho_{2} u ; \quad d_{1}=k_{3} \rho_{2} u ;$

$$
\begin{aligned}
& s_{1}(u)=-u J_{1}(u)-B i J_{0}(u) \\
& s_{2}(u)=-u Y_{1}(u)-B i Y_{0}(u)
\end{aligned}
$$

$S_{0}(u)=s_{1}(u) Y_{0}\left(\bar{a}_{1}\right)-s_{2}(u) J_{0}\left(\bar{a}_{1}\right) ;$

$$
S_{1}(u)=s_{1}(u) Y_{1}\left(\bar{a}_{1}\right)-s_{2}(u) J_{1}\left(\bar{a}_{1}\right) \text {; }
$$$$
U_{1}(u)=Y_{1}(b) J_{0}\left(c_{1}\right)-J_{1}\left(b_{1}\right) Y_{0}\left(c_{1}\right)
$$$$
U_{2}(u)=J_{0}\left(b_{1}\right) Y_{0}\left(c_{1}\right)-Y_{0}\left(b_{1}\right) J_{0}\left(c_{1}\right)
$$

$$
U_{3}(u)=Y_{1}\left(b_{1}\right) J_{1}\left(c_{1}\right)-J_{1}\left(b_{1}\right) Y_{1}\left(c_{1}\right)
$$$$
U_{4}(u)=J_{0}\left(b_{1}\right) Y_{1}\left(c_{1}\right)-Y_{0}\left(b_{1}\right) J_{1}\left(c_{1}\right)
$$

Here $J_{0}(z), Y_{0}(z), J_{1}(z), Y_{1}(z)$ - zero-order and first-order order Bessel functions of the first and second type [6]-[9].

Calculating the non-real integrals, which are contained in formula (37), dimensionless temperature of the pipe housing is obtained.

\section{PiPe Housing InSUlation TEMPERATURE CALCUlation}

The original pipe housing insulation temperature is expressed in [1]-[3] using formulas (24) and (35) with an integral as follows:

$\Theta_{2}(\rho, F)=\Theta_{02}+\frac{1}{2 \pi j} \int_{\gamma-j \omega}^{\gamma+j \omega} \mathrm{e}^{p F} \frac{\Delta C_{3}(\sqrt{p})}{\Delta(\sqrt{p})} I_{0}\left(k_{2} \rho \sqrt{p}\right) \mathrm{d} p$

$$
+\frac{1}{2 \pi j} \int_{\gamma-j \omega}^{\gamma+j \omega} \mathrm{e}^{p F} \frac{\Delta C_{4}(\sqrt{p})}{\Delta(\sqrt{p})} K_{0}\left(k_{2} \rho \sqrt{p}\right) \mathrm{d} p
$$

The calculation of complex-variable integrals is discussed in monographs [1]-[3] and publications [9], [10].

Expressing complex-variable function integrals in a realistic manner, with dimensionless expression of pipe insulation temperature $\left(\rho_{1} \leq \rho \leq \rho_{2}\right)$ the following formula is obtained:

$$
\begin{gathered}
\Theta_{2}(\rho, F)=1+\left(\Theta_{02}-\Theta_{01}\right)-\left(\frac{2}{\pi}\right)^{2}\left(\Theta_{01}-1\right) \Lambda_{1} \frac{B i}{\rho_{1}^{2}} \\
\cdot\left[\int_{0}^{+\infty} \mathrm{e}^{-u^{2} F} \frac{\phi(u) \psi_{3}(u)+\psi(u) \phi_{3}(u)}{\phi^{2}(u)+\psi^{2}(u)} J_{0}\left(k_{2} \rho u\right) \frac{\mathrm{d} u}{u^{2}}\right. \\
\left.-\int_{0}^{+\infty} \mathrm{e}^{-u^{2} F} \frac{\phi_{4}(u) J_{0}\left(\rho k_{2} u\right)-\psi_{4}(u) Y_{0}\left(\rho k_{2} u\right)}{\phi^{2}(u)+\psi^{2}(u)} J_{0}\left(k_{2} \rho u\right) \frac{\mathrm{d} u}{u^{2}}\right] .
\end{gathered}
$$

where

$$
\begin{aligned}
& \phi_{3}(u)=k_{3}[\left.J_{0}\left(c_{1}\right) J_{1}\left(d_{1}\right)-Y_{0}\left(c_{1}\right) Y_{1}\left(d_{1}\right)\right] \\
&+\Lambda_{2} k_{2}\left[J_{1}\left(c_{1}\right) J_{0}\left(d_{1}\right)-Y_{1}\left(c_{1}\right) Y_{0}\left(d_{1}\right)\right] ; \\
& \psi_{3}(u)=k_{3}\left[Y_{0}\left(c_{1}\right) J_{1}\left(d_{1}\right)+J_{0}(c) Y_{1}\left(d_{1}\right)\right] \\
&+\Lambda_{2} k_{2}\left[J_{1}\left(c_{1}\right) Y_{0}\left(d_{1}\right)+Y_{1}\left(c_{1}\right) J_{0}\left(d_{1}\right)\right] ; \\
& \phi_{4}(u)= k_{3} J_{0}\left(\bar{a}_{1}\right) Y_{1}\left(d_{1}\right)+\Lambda_{2} k_{2} J_{1}\left(c_{1}\right) Y_{0}\left(d_{1}\right) ; \\
& \psi_{4}(u)=k_{3} J_{0}\left(\bar{a}_{1}\right) J_{1}\left(d_{1}\right)+\Lambda_{2} k_{2} J_{1}\left(c_{1}\right) J_{0}\left(d_{1}\right) .
\end{aligned}
$$

Calculating the improper integrals in formula (54), the dimensionless temperature of the pipe insulation is obtained.

\section{SOIL TEMPERATURE CALCUlation}

The original environment temperature is expressed in [1]-[3] using formulas (25) and (35) with an integral as follows:

$$
\begin{gathered}
\Theta_{3}(\rho, F)=\Theta_{03} \\
+\frac{1}{2 \pi j} \int_{\gamma-j \omega}^{\gamma+j \omega} \mathrm{e}^{p F} \frac{\Delta C_{5}(\sqrt{p})}{\Delta(\sqrt{p})} K_{0}\left(k_{3} \rho \sqrt{p}\right) \mathrm{d} p
\end{gathered}
$$

The integral in formula (59) is calculated as described above. Expressing complex-variable function integrals in a realistic manner, with dimensionless expression of environment temperature $\left(\rho \geq \rho_{2}\right)$ the following formula is obtained:

$$
\begin{gathered}
\Theta_{3}(\rho, F)=\Theta_{03}+\left(1-\Theta_{01}\right) \frac{\rho_{1}}{\rho_{2}}+\left(\frac{2}{\pi}\right)^{3}\left(\Theta_{01}-1\right) \Lambda_{1} \Lambda_{2} \\
\cdot \frac{B i}{\rho_{2}^{2}} \int_{0}^{+\infty} \mathrm{e}^{-u^{2} F} \frac{\phi(u) J_{0}\left(k_{3} \rho u\right)-\psi(u) Y_{0}\left(k_{3} \rho u\right)}{\phi^{2}(u)+\psi^{2}(u)} \frac{\mathrm{d} u}{u^{3}} .
\end{gathered}
$$


Calculating the non-real integrals in formula (54), environmental dimensionless environment temperature is obtained.

\section{CONCLUSION}

As there is no unified widely accepted methodology for the determination of heat flow between a heat source including the surrounding environment and the heat/cold user, the methodology offered is intended for bringing heat flow calculations nearer to the actual situation. This methodology can be used by heat technology specialists for designing, planning and other purposes.

\section{REFERENCES}

[1] H. S. Carslaw and J. C. Jaeger, Teploprovodnostj tverdih tel. Moscow: Nauka, 1964, 487 p. (in Russian)

[2] H. S. Carslaw and J. C. Jaeger, Operacionnie metodi v prikladnoj matematike, Moscow: IL, 1948, 293 p. (in Russian)

[3] M. A. Lavrentjev and B. V. Šabat, Metodi teorii funkcij kompleksnogo peremennogo, Moscow: Nauka, 1958, 678 p. (in Russian)

[4] V. Barkāns, A. Cers, N. Zeltinsh, and G. Turlajs, "Non-Stationary Temperature Field in a Tube Surrounding Environment," Modern science: researches, ideas, results, technologies, no.2(13), Dnepropetrovsk, Ukraine, NPVK Triakon, pp. 34-37, 2013.

[5] V. Barkāns, "Operator Method for Nonstationary Temperature Field Calculation in Thick-Walled Cylindrical Heat Pipe Environment," Power and Electrical Engineering, vol. 33, pp. 5-11, 2016. https://doi.org/10.7250/pee.2016.001

[6] G. N. Watson, Teorija besselevih funkcij. Moscow: IL, 1949, 787 p. (in Russian)

[7] G. Doetsch, Rukovodstvo k prakticheskomu primeneniju preobrazovanija Laplassa i Z-preobrazovanija, Moskva: Nauka, 1971, 288 p. (in Russian)

[8] A. Gray and G. B. Matthews, Funkcii Besselja $i$ ih prilozhenija $k$ fizike $i$ mehanike, Moscow: IL, 1953, 372 p. (in Russian)

[9] E. Janke, F. Emde, and F. Losch, Specialjnije funkcii, formuli, grafiki, tablici, Moscow: Fizmat-giz, 1968, 344 p. (in Russian)

[10] V. P. Isachenko, V. A. Osipova, and A. S. Sukomel, Teploperedacha, Moscow: Energija, 1969, 440 p. (in Russian)

[11] V. Katsura, K. Nagano, and S. Takeda, "Method of calculation of the ground temperature for multiple ground heat exchangers," in Proc. Applied Thermal Engineering, vol. 28, no. 14-15, pp. 1995-2004, 2008. https://doi.org/10.1016/j.applthermaleng.2007.12.013 